\title{
Murciélagos de cola corta (Carollia: Phyllostomidae) del Parque Nacional Natural Gorgona (Colombia) y sus implicaciones biogeográficas
}

\author{
Oscar E. Murillo-García \\ Departamento de Biología. Universidad del Valle, Cali, Colombia; oscar.murillo@correounivalle.edu.co
}

\author{
Recibido 18-X-2013. Corregido 20-XI-2013. Aceptado 19-XII-2013.
}

\begin{abstract}
Short-tailed bats (Carollia: Phyllostomidae) from Gorgona National Natural Park (Colombia) and its biogeographical implications. Taxonomy of short-tailed bats (Phyllostomidae: Carollia) has been unclear due to the extreme morphological similarity among species and the significant morphological variation within species. The identity of the Carollia species in Gorgona National Natural Park (Colombian Pacific) has been controversial due to the high morphological similarity between Carollia perspicillata and C. brevicauda. C. perspicillata is common in lowlands, and more likely to be the Gorgona species than C. brevicauda, while geological evidence suggests that common between 1500 and $3000 \mathrm{~m}$. We recorded eight measurements from jaws and skulls of confirmed $C$. perspicillata (24 individuals) and C. brevicauda (23) and 35 individuals captured in Gorgona. Discriminant analyses showed that, contrary to expectations based on current altitudinal distributions, the Gorgona population is morphologically closer to C. brevicauda. Biological evidence suggests that Gorgona was connected in the past to mainland South America. Gorgona may be the highest part of a now submerged mountain range ("Cordillera de la Costa") that was part of the continent during the Pleistocene glaciations. Consequently, I hypothesize that $C$. brevicauda colonized Gorgona overland during last Pleistocene glaciations. Rev. Biol. Trop. 62 (Suppl. 1): 435-445. Epub 2014 February 01.
\end{abstract}

Key words: bat dispersal, cranial morphology, island colonization, biogeography, bat taxonomy.

Los murciélagos de cola corta (Carollia, Gray 1838: Phyllostomidae, Gray 1825) se caracterizan por presentan extensas distribuciones geográficas y una gran abundancia local, por lo cual están entre los mamíferos más comunes de la fauna neotropical. Sin embargo, la taxonomía del género ha sido imprecisa debido a la dificultad para diferenciar adecuadamente entre las especies. Pine (1972) advirtió sobre la variación que existe dentro de las especies del genero, mientras McLellan (1984) cuantificó esta variación y encontró diferencias intra-específicas significativas en morfometría craneal en todas las especies. Este patrón complejo de variación morfológica dificulta la diferenciación entre algunas especies del género. El estatus sistemático de las especies de Carollia se mantuvo sin modificar durante gran tiempo después de la revisión de Pine (1972); hasta la descripción de nuevas especies (Baker, Solari \& Hoffmann, 2002; Muñoz, Cuartas-Calle \& González, 2004; Pacheco, Solari \& Velazco, 2004; Solari \& Baker, 2006). Recientemente, se reconocieron ocho especies para el género (McLellan \& Koopman, 2007), cuatro de las cuales se encuentran en Colombia (Zurc \& Velasco 2010): Carollia perspicillata (Linnaeus 1758), Carollia brevicauda (Schinz 1821), Carollia monohernandezi Muñoz et al. 2004 y Carollia castanea Allen 1890.

En su revisión del genero Carollia, Pine (1972) presentó una combinación de caracteres para distinguir entre las especies. Adicionalmente, afirmó que aunque los individuos de la mayoría de las áreas de simpatría pueden ser asignados correctamente a una especie, algunos 
individuos de poblaciones alopátricas parecen ser morfológicamente intermedios entre especies; y pueden ser asignados erróneamente a una especie con base en los caracteres comúnmente utilizados para diferenciarlas. Por lo tanto, es difícil encontrar caracteres distintivos que consistentemente separen las especies del género a lo largo de sus rangos de distribución (Pine, 1972; McLellan, 1984; Owen, Schmidly \& Davis, 1984; Zurc \& Velasco, 2010). Consecuentemente, los caracteres propuestos para diferenciar entre C. brevicauda y C. perspicillata, las especies más comunes del género, son difíciles de utilizar en muchas ocasiones (Pine, 1972; McLellan, 1984; Owen et al., 1984; York \& Papes, 2007; Zurc \& Velasco, 2010). En Colombia la identificación de estas especies se ha dificultado debido a que estas presentan un amplio traslape en sus distribuciones geográficas y un alto grado de variación morfológica.

En el Parque Nacional Natural Gorgona (PNN Gorgona, Colombia), la identidad de la especie del género Carollia que habita la isla ha sido controversial. Algunos autores consideraron que los murciélagos de cola corta de la isla corresponden a $C$. perspicillata (Thomas, 1926; Cadena, Gómez-Laverde, Andrade \& Peñuela, 1990), mientras Alberico (1986a; 1987) consideró que la especie de la isla es C. brevicauda. De acuerdo con los rangos de distribución altitudinal actuales, se espera que C. perspicillata sea la especie que habita la isla debido a que se distribuye en las tierras bajas sobre la costa pacífica colombiana; mientras que $C$. brevicauda se distribuye sobre la cordillera de los Andes, usualmente en alturas por encima de $1000 \mathrm{~m}$. Recientemente, la población de Gorgona se ha considerado como una localidad marginal para la distribución geográfica de C. perspicillata (McLellan \& Koopman, 2007). Sin embargo, Thomas (1926) y Cadena et al. (1990) basaron sus identificaciones en un solo espécimen, por lo cual debido a la gran variación morfológica intra-específica y la alta similitud entre las especies del género, esta identificación no es concluyente. Previamente se ha encontrado que $C$. perspicillata y C. brevicauda se pueden diferenciar con base en morfometría clásica a partir de diferentes caracteres morfológicos craneales (McLellan, 1984; Owen et al., 1984; York \& Papes, 2007; Zurc \& Velasco, 2010). Por lo tanto, debido a la dificultad para distinguir entre las especies de Carollia con base en caracteres cualitativos, se realizó un estudio de morfometría craneal para determinar la identidad de los murciélagos del género Carollia que habitan en el PNN Gorgona. La correcta identificación de estos murciélagos es importante pues el conocimiento de la identidad de las especies y su distribución geográfica, especialmente en las áreas protegidas, es importante para implementar las iniciativas de preservación y utilización de la biodiversidad (Heywood \& Watson, 1997).

\section{MATERIALES Y MÉTODOS}

Se seleccionaron un total de 82 individuos: 24 de Carollia brevicauda, 23 de Carollia perspicillata y 35 de la población de Gorgona. La mayoría de los especímenes provienen de la colección de Mamíferos del la Universidad del Valle (UV), con excepción de ocho individuos que están depositados en el Instituto de Ciencias Naturales (ICN) de la Universidad Nacional de Colombia, Bogotá. Se midieron ocho caracteres morfológicos, los cuales se seleccionaron con base en características cuantitativas y cualitativas usadas tradicionalmente para la separación de las especies $C$. perspicillata y $C$. brevicauda: longitud mayor del cráneo (LC), longitud de la hilera dental superior (HDS), longitud de la hilera dental inferior (HDI), longitud cóndilo-basal (LCB), longitud mandibular (LM), ancho de la constricción post-orbital (C), profundidad mandibular (PM) y alto del cráneo $(\mathrm{AC})$. Las mediciones se realizaron en milímetros con un calibrador digital.

Análisis de datos: Con el fin de asignar los individuos del PNN Gorgona a la especie correcta, se realizó un análisis discriminante utilizando los caracteres morfológicos considerados en las mediciones y dos grupos correspondientes a las especies. El análisis discriminante produce funciones de clasificación 
para cada grupo, a través de la combinación lineal de las variables utilizadas (McCune \& Grace, 2002), las cuales se utilizaron para asignar los individuos problema (Gorgona) a los grupos definidos a priori (C. perspicillata y $C$. brevicauda). Adicionalmente, se realizó un análisis discriminante con tres grupos a priori (C. perspicillata, C. brevicauda y Gorgona), con el fin de evaluar el grado de diferenciación morfológica entre estos. Para los análisis discriminantes se consideraron todas las medidas morfológicas y se realizaron validaciones cruzadas, dejando un individuo por fuera al tiempo (Lachenbruch \& Mickey, 1968), con el fin de evaluar la confiabilidad de los resultados. Esta validación consiste en realizar análisis discriminantes en los cual se deja por fuera una observación, y luego se utilizan las funciones discriminantes resultantes para clasificar la observación dejada por fuera (Lachenbruch \& Mickey, 1968). Posteriormente, se evaluó la diferenciación morfológica entre los grupos a través de la comparación de las distancias cuadradas de Mahalanobis entre los grupos (McCune \& Grace, 2002), para lo cual se tomaron como referencia los centroides de los grupos.

Las comparaciones entre los grupos se realizaron con base en la relación logarítmica (log-ratio), la cual es una medida del tamaño del efecto (effect size) y por lo tanto es independiente de los tamaños de muestra de los grupos que se están comparando (Hedges, Gurevitch \& Curtis 1999; Nakagawa \& Cuthill, 2007). Las relaciones logarítmicas relacionaron las distancias dentro de los grupos (entre los individuos de un grupo y su centroide) con las distancias entre grupos (desde el centroide de un grupo hasta los individuos de otro grupo) de la siguiente forma (Hedges et al., 1999):

$$
\rho_{i j}=\ln \left[\frac{d_{i j}}{d_{i i}}\right]
$$

donde $\rho_{i j}$ es la relación logarítmica entre los grupos $i$ y $j$ medida desde el centroide del grupo $i ; \mathrm{d}_{i i}$ es la distancia cuadrada de Mahalanobis promedio desde el centroide $i$ hasta los individuos del mismo grupo $\mathrm{y}_{i j}$ es la distancia desde el centroide $i$ hasta los individuos del grupo $j$. Por lo tanto, esta medida permite estimar la magnitud de la diferencia morfológica entre los dos grupos considerados, sin incluir el efecto de las diferencias en los tamaños de las muestras de los grupos comparados. La varianza de la relación logarítmica fue obtenida a partir del método delta (Bolker, 2008) como:

$$
\sigma\left(\rho_{i j}\right)=\frac{1}{n_{i i}}\left[\frac{\sigma\left(d_{i j}\right)}{d_{i i}^{2}}\right]+\frac{1}{n_{i j}}\left[\frac{\sigma\left(d_{i j}\right)}{d_{i i}}\right]
$$

$\sigma\left(\rho_{i j}\right)$ es la varianza de la relación logarítmica entre los grupos $i$ y $j$ medidos desde el centroide del grupo $i, \sigma_{(d i i)}$ es la varianza de las distancias cuadradas de Mahalanobis de los individuos del grupo $i$ hacia el centroide del grupo $i, \sigma_{(d i j)}$ es la varianza de las distancias cuadradas de Mahalanobis de los individuos del grupo $i$ hacia el centroide del grupo $j, \mathrm{~d}_{i i}$ es la distancia cuadrada de Mahalanobis promedio desde el centroide $i$ hasta los individuos del mismo grupo $\mathrm{y} \mathrm{d}_{i j}$ es la distancia desde el centroide $i$ hasta los individuos del grupo $j$. Para evaluar si las diferencias en morfología entre los grupos fueron significativas, se calcularon intervalos de confianza del $95 \%$ con base en los valores de la relación logarítmica y su varianza. Los análisis discriminantes fueron realizados por medio del programa R 2.14 (R Development Core Team, 2012).

\section{RESULTADOS}

El análisis discriminante produjo una sola función, la cual reveló una asociación significativa entre los grupos y las variables predictoras, y contuvo el $100 \%$ de la variabilidad entre los grupos. Las distribuciones de los valores de la función discriminante para las dos especies no se traslaparon entre si, lo cual indicó una discriminación significativa entre estas a partir el análisis realizado (Fig. 1). La clasificación a posteriori del análisis discriminante y la validación cruzada clasificaron correctamente todos los individuos de Carollia perspicillata y C. brevicauda, resultando en un $100 \%$ de concordancia con la clasificación 
a priori. Las variables más importante para la discriminación entre las dos especies fueron las longitudes de las hileras dental superior e inferior (coeficientes de la función discriminante: $\mathrm{HDS}=5.00$, HDI=3.22), las cuales fueron significativamente menores para $C$. brevicauda en comparación con C. perspicillata (Cuadro 1). La evaluación de las funciones de clasificación indicó que los individuos de Gorgona deben ser asignados a $C$. brevicauda, porque ellos presentan valores en el rango correspondiente a esta especie.

Los resultados del segundo análisis discriminante revelaron que existe una discriminación morfológica entre los tres grupos considerados (Fig. 2). La clasificación a posteriori del análisis discriminante clasificó el $87.5 \%$ de los individuos de acuerdo a su asignación a priori $(83.3 \%$ brevicauda, $100.0 \%$ perspicillata, $91.4 \%$ de Gorgona), mientras el procedimiento de validación cruzada clasificó correctamente $87.8 \%$ de los individuos $(83.3 \%$ de C. brevicauda, 100,0\% de C. perspicillata, $82.9 \%$ de Gorgona). Las discrepancias se debieron a la clasificación de algunos especímenes de $C$. brevicauda como pertenecientes a la población de Gorgona y viceversa. La primera función discriminante contuvo el $89.1 \%$ de la variabilidad entre los grupos, y sobre este eje los individuos de Gorgona y de C. brevicauda

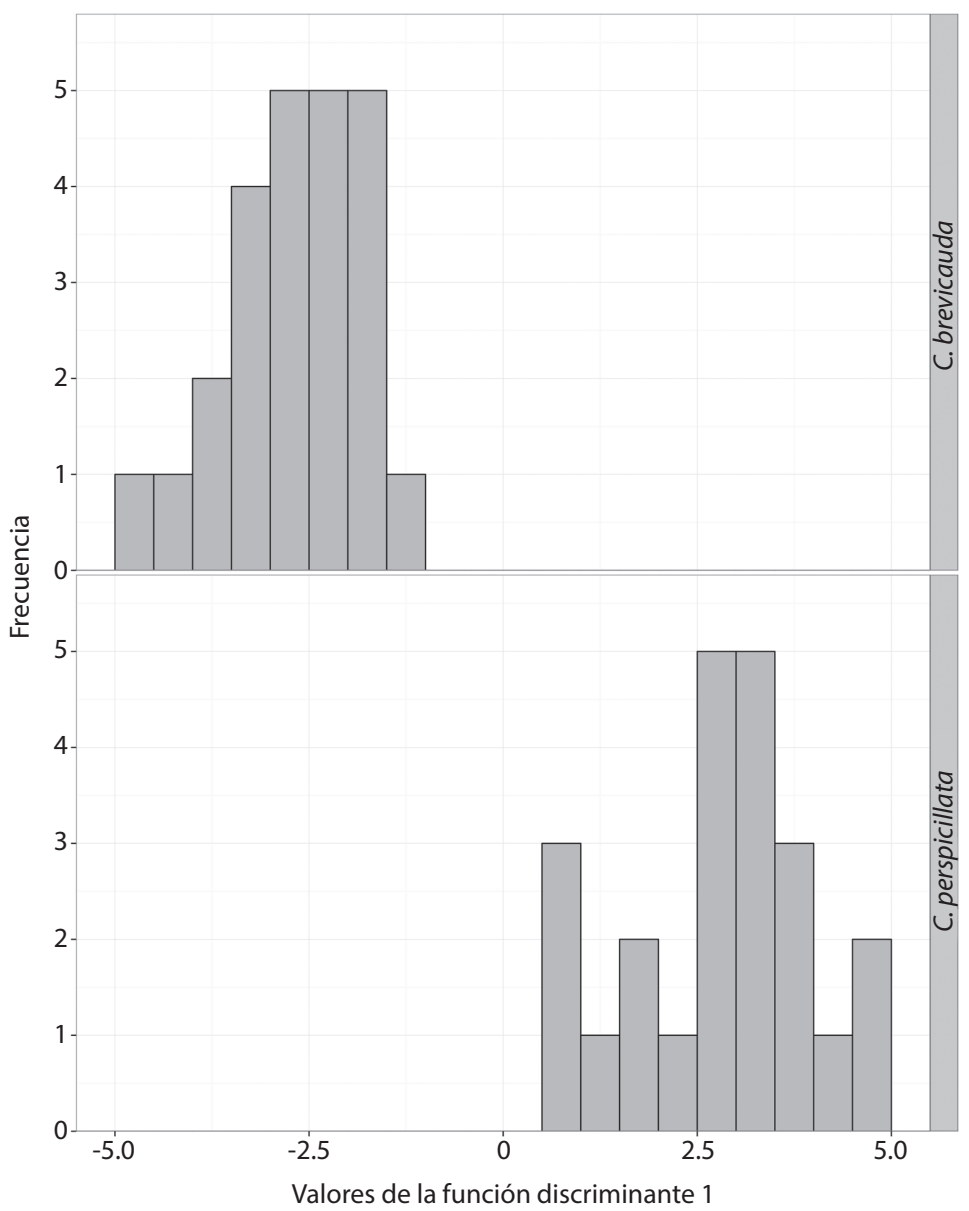

Fig. 1. Histogramas mostrando la distribución de los valores de la función discriminante 1 para las especies Carollia brevicauda y $C$. perspicillata.

Fig. 1. Histograms showing the distribution of discriminant scores from function 1 for Carollia brevicauda and $C$. perspicillata. 
CUADRO 1

Media ( \pm érror estándar) de la mediciones morfológicas realizadas a individuos de C. brevicauda, C. perspicillata y de la población de Isla Gorgona

TABLE 1

Mean ( \pm standard error) of morphological measurements of individuals of C. brevicauda, C. perspicillata and Gorgona National Natural Park

\begin{tabular}{cccc} 
Medición & C. brevicauda & C. perspicillata & PNN Gorgona \\
LC & $22.40 \pm 0.06$ & $23.10 \pm 0.10$ & $22.60 \pm 0.07$ \\
LCB & $20.60 \pm 0.05$ & $21.10 \pm 0.10$ & $20.70 \pm 0.07$ \\
AC & $11.10 \pm 0.07$ & $11.20 \pm 0.07$ & $10.50 \pm 0.14$ \\
C & $5.40 \pm 0.03$ & $5.70 \pm 0.03$ & $5.60 \pm 0.03$ \\
LM & $14.40 \pm 0.05$ & $14.80 \pm 0.06$ & $14.50 \pm 0.05$ \\
PM & $2.20 \pm 0.04$ & $2.40 \pm 0.03$ & $2.30 \pm 0.04$ \\
HDS & $7.00 \pm 0.04$ & $7.70 \pm 0.04$ & $6.90 \pm 0.03$ \\
HDI & $7.70 \pm 0.03$ & $8.40 \pm 0.04$ & $7.60 \pm 0.03$ \\
\hline
\end{tabular}

LC: Longitud mayor del cráneo; LCB: longitud cóndilo-basal; AC: alto del cráneo; C: ancho de la constricción post-orbital; PM: profundidad mandibular; LM: Longitud mandibular; HDS: longitud de la hilera dental superior; HDI: longitud de la hilera dental inferior.

LC: greatest length of skull; LCB: condyle basal length; AC: height of braincase; C: post-orbital constriction width; PM: mandibular depth; LM: mandibular length; HDS: length of maxillary tooth row; (HDI): length of mandibular tooth row.

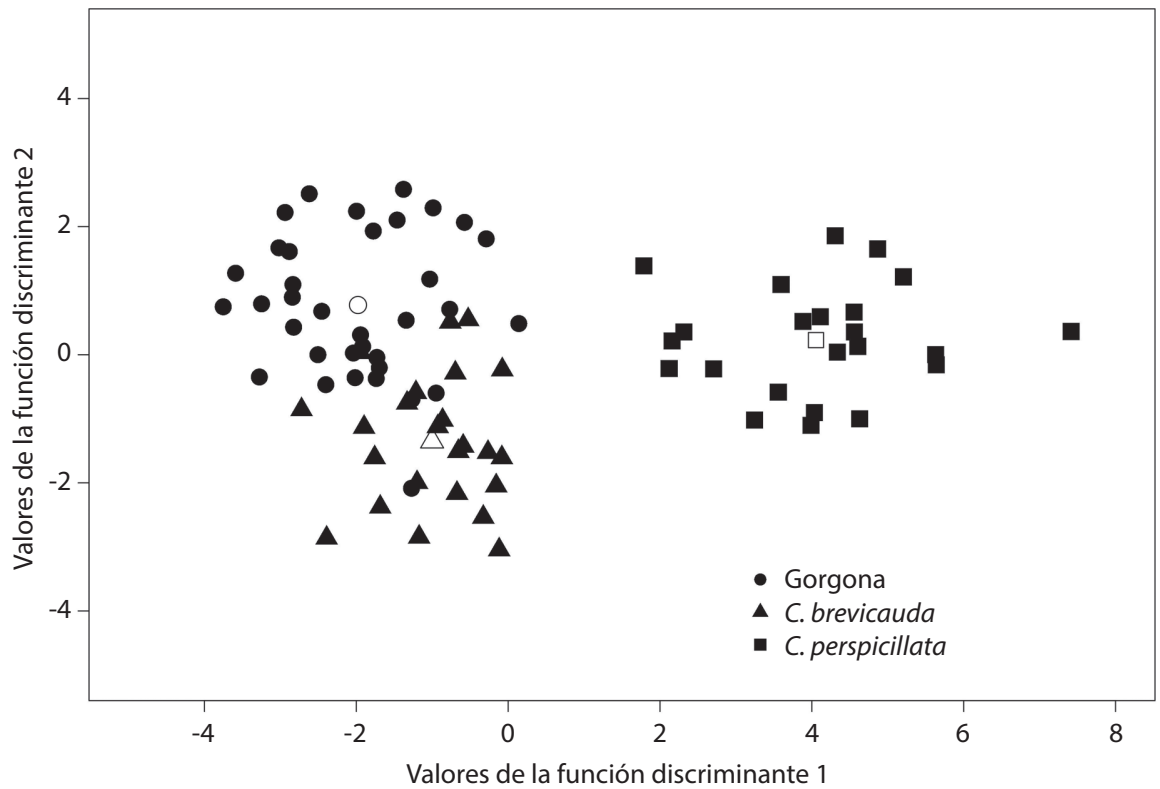

Fig. 2. Comparación morfológica entre las poblaciones de C. brevicauda, C. perspicillata y la población del PNN Gorgona. Los simbolos sin rellenar representan los centroides de los grupos respectivos.

Fig. 2. Morphological comparison between populations of C. brevicauda, C. perspicillata, and Gorgona National Park. Empty symbols represent the centroids for each group. 
se diferenciaron de los de C. perspicillata. Por otra parte, el $10.9 \%$ de la variabilidad entre los grupos contenida en la segunda función discriminante permitió diferenciar entre la población de Gorgona y los individuos de C. brevicauda (Fig. 2). Las longitudes de las hileras dentales fueron la características más importantes para distinguir entre $C$. perspicillata y $C$. brevicauda (coeficientes de la primera función discriminante: $\mathrm{HDS}=3.59$, $\mathrm{HDI}=4.16$ ), mientras el ancho de la constricción post-orbital fue la característica más importante para discriminar entre la población de Gorgona y C. brevicauda (coeficientes de la segunda función discriminante: $\mathrm{C}=5.30$ ) (Cuadro 1).

Los intervalos de confianza (95\%) para la relación logarítmica no incluyen cero, lo cual sugiere que los grupos se diferencian morfológicamente entre si (Fig. 3). Adicionalmente, las relaciones logarítmicas entre la población de Gorgona y C. brevicauda fueron significativamente menores de lo que fueron con $C$. perspicillata (Fig. 3). Por lo tanto, este resultado sugiere que aunque todas las poblaciones son diferentes entre si, la población de Gorgona es más similar morfológicamente a $C$. brevicauda que a $C$. perspicillata.

\section{DISCUSSION}

A pesar de la gran cantidad de especímenes en las colecciones zoológicas, la taxonomía del género Carollia es confusa debido a la dificultad para diferenciar adecuadamente entre las especies. Particularmente, Carollia brevicauda y Carollia perspicillata son extremadamente similares en tamaño y morfología, y sus rangos de distribución se traslapan en diversas localidades; lo cual ha causado dificultades en la identificación de algunos especímenes en ciertas áreas (Pine, 1972; McLellan, 1984; Zurc \& Velasco, 2010). La dificultad para distinguir entre estas especies puede ser atribuida a la alta variación intra-específica, aunque se ha sugerido que hibridación y convergencia de caracteres pueden ser factores adicionales

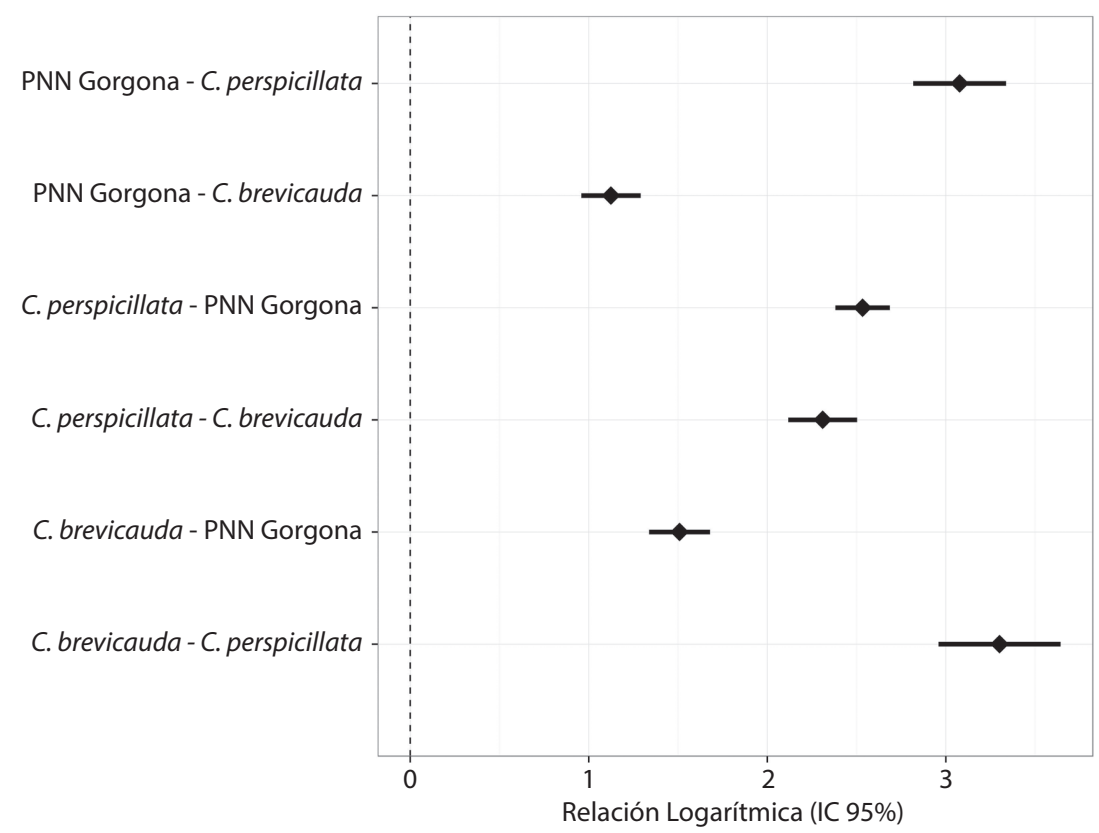

Fig. 3. Intervalos de confianza (95\%) para las relaciones logarítmicas de las comparaciones entre C. brevicauda, $C$. perspicillata y los individuos de PNN Gorgona.

Fig. 3. Confidence intervals (95\%) for log-ratio comparisons among C. brevicauda, C. perspicillata and individuals from PNN Gorgona. 
que complican la delimitación de estas especies (McLellan, 1984). El primer reporte de un ejemplar del género Carollia en el parque Nacional Natural Gorgona fue realizado por Thomas (1926), quien identificó una hembra como C. perspicillata. Posteriormente, Alberico $(1986 ; 1987)$ con base en una serie de individuos más grande (14 especímenes), consideró que la especie residente en la isla es $C$. brevicauda. Por otra parte, Cadena et al. (1990) con base en mediciones de la longitud de la tibia, la longitud de la serie dental superior y la longitud de la serie dental inferior de una hembra, consideró que la especie presente en Gorgona es C. perspicillata. Sin embargo, de las tres características morfométricas evaluadas una correspondió a $C$. brevicauda, otra a $C$. perspicillata, y la otra se encontró en el rango de superposición de las dos especies.

El resultado del análisis morfométrico, del presente estudio, reveló que C. brevicauda y C. perspicillata pueden ser distinguidas con base en las longitudes de las hileras dentales, la cual son menores para $C$. brevicauda (C. brevicauda: $\mathrm{HDS}=$ media $7.00 \mathrm{~mm}[$ mínimo 6.72-máximo 7.20], HDI=7.67mm [7.34-7.89]; C. perspicillata: $\mathrm{HDS}=7.71 \mathrm{~mm}$ [7.31-8.37], HDI $=8.42 \mathrm{~mm}$ [8.01-9.20]). Los resultados de los análisis discriminantes son confiables pues las clasificaciones a posteriori y las validaciones cruzadas no mostraron discrepancias grandes con respecto a las clasificaciones $a$ priori. Esto índica que las variables utilizadas en el presente análisis tienen suficiente poder discriminatorio para diferenciar entre las especies. Lo cual probablemente se deba a que en la escogencia de las variables utilizadas se consideraron características que se han propuesto para la separación de las especies en cuestión (ver Pine, 1972; McLellan, 1984).

Los resultados del presente estudio indican que la especie presente en el PNN Gorgona es morfológicamente más similar a $C$. brevicauda que a $C$. perspicillata. Estos resultados contradicen las predicciones basadas en las diferencias actuales entre los rangos de distribución altitudinal de estas especies. C. perspicillata es la especie con mayor probabilidad de habitar en la isla debido a que actualmente se distribuye en las tierras bajas de la costa pacífica colombiana, mientras $C$. brevicauda es una especie predominantemente de tierras altas. Por lo tanto, surge el interrogante de cómo C. brevicauda colonizó Gorgona a partir de poblaciones continentales alejadas de la costa y sobre la cordillera de los Andes.

Implicaciones biogeográficas: Es poco probable que $C$. brevicauda colonizara la isla directamente desde el continente, pues este tipo de eventos de dispersión están restringidos para los murciélagos ya que difícilmente toleran hambre y son altamente susceptibles a la desecación (Silva-Taboada, 1974). Sin embargo, los cambios en el nivel del mar constituyen un mecanismo viable para facilitar la dispersión entre masas de tierra. En particular los periodos de nivel del mar excepcionalmente bajos han facilitado la dispersión de murciélagos al disminuir la separación entre masas terrestres (Presley \& Willig, 2008).

Gorgona es una isla continental y como tal su historia biogeográfica está ligada a la del continente, por tanto para entender la composición de la biota presente en esta isla es necesario considerar su historia geológica (Alberico, 1986b). Durante el Eoceno se formó un rango montañoso que bordeaba la depresión que representa la última conexión entre los océanos Atlántico y Pacífico, pasando por el límite noroeste de Sudamérica (Gansser, 1950; Nygren, 1950; Acosta, 1986). Esta cordillera denominada "Cordillera de la Costa" (Acosta, 1986) actualmente está sumergida entre Cabo Corrientes y Esmeraldas, siendo Gorgona la única parte que alcanza una altura suficiente para ser isla, y de ella hacen parte la Serranía de los Saltos y la Serranía del Baudó en el continente (Haffer, 1970; Acosta, 1986; Alberico, 1986b). Sin embargo, la existencia de Gorgona como isla ha dependido de las fluctuaciones del nivel del mar que se presentaron durante el pleistoceno (2.59 millones de años hasta aproximadamente 11700 años atrás). Se considera que, por la poca profundidad del mar que la separa del continente, Gorgona 
ha formado parte del continente durante los periodos glaciares y ha retornado a ser isla con el aumento del nivel del mar durante los periodos interglaciares (Alberico, 1986b). Sin embargo, esta conexión no explica la presencia de C. brevicauda en la Isla, pues con base en las distribuciones altitudinales actuales es probable que las poblaciones de C. brevicauda se encontraran más alejadas de Gorgona que las de $C$. perspicillata.

En la isla se encuentran especies que revelan una afinidad de la biota de Gorgona con los andes y las tierras bajas hacia el sur de la isla (sur de Colombia-norte de Ecuador). En Gorgona, se encuentra una especie de árbol conocido como pino Colombiano (Podocarpus oleifolius), la cual se distribuye en el occidente de Colombia y es típica del bosque nublado (Alberico, 1986b). Igualmente, en la isla se ha descrito una especie de cangrejo de agua dulce, Hypolobocera gorgonensis, que pertenece a un grupo de especies que se encuentra asociado a zonas montañosas de los Andes (von Prahl, 1983). La presencia de estas especies, y de $C$. brevicauda, se ha sugerido como evidencia a favor de la conexión entre la isla y el continente (Alberico 1986b), pero además sugiere que pudo existir una conexión entre Gorgona y los andes en el pasado. Por otra parte, aunque la fauna terrestre guarda una estrecha relación con la fauna continental, algunos organismos tienen más relación con especies de la costa pacífica del sur de Colombia - norte de Ecuador que con especies de la costa en frente de la isla. Algunas especies de saurios tienen mayor parentesco con las especies localizadas hacia el sur de Colombia (Ayala, Carvajal, Caro \& Castro, 1979). Igualmente, las especies más cercanas para dos especies de escorpiones reportadas para Gorgona, se encuentran en la zona entre Tumaco en Colombia y Esmeraldas en Ecuador (Lourenço \& Flores, 1989); y una especie de escarabajo del Género Boatia (Coleoptera: Mordellidae) capturada en Gorgona, representa el primer registro del género fuera del Ecuador (Martínez, 2001).

Por lo tanto, con base en la historia geológica y en las afinidades de la biota de Gorgona con el continente, es probable que haya existido una ruta de colonización para la fauna a través de la cordillera a la cual pertenece Gorgona. Esta conexión pudo presentarse hacia el sur de la isla como consecuencia del descenso en el nivel del mar que probablemente resultó en la exposición de la cordillera a la cual pertenece Gorgona (Fig. 4).

Análisis filogenéticos, filogeográficos y de coalescencia han concluido que $C$. perspicillata y $C$. brevicauda tienen un tiempo de origen similar hace aproximadamente 700000 años, sugiriendo que estas especies se originaron simultáneamente a partir de eventos vicariantes durante el pleistoceno (Hoffmann \& Baker, 2003; Pavan \& Martins, 2011). Adicionalmente, el patrón de diferenciación intra-específico indica que las subdivisiones filogeográficas de estas especies pueden estar relacionados con los cambios asociados a las oscilaciones climáticas del pleistoceno (Hoffmann \& Baker, 2003; Pavan \& Martins, 2011). Por lo tanto, esta evidencia sugiere que la distribución actual de C. brevicauda ha sido afectada en el pasado por los eventos climáticos del pleistoceno. Lo cual es congruente con la hipótesis de colonización de esta especie en Gorgona planteada en el presente estudio. Por lo tanto, existe evidencia geológica, biológica y biogeográfica que apoya la hipótesis de una ruta de colonización de C. brevicauda al PNN Gorgona por medio de una conexión con los Andes a través de la Cordillera de la Costa; proceso que pudo facilitarse por el descenso en el nivel de los océanos durante las glaciaciones del pleistoceno. Sin embargo, es necesario evaluar esta hipótesis a partir de análisis moleculares y filogeográficos considerando, además, otros grupos de organismos terrestres.

\section{AGRADECIMIENTOS}

A Michael Alberico por su enseñanza y apoyo. A María Cleopatra Pimienta y María Juliana Bedoya-Durán por su colaboración con las mediciones de los especímenes y la discusión de las ideas expuestas en el documento. Finalmente, agradezco a dos 


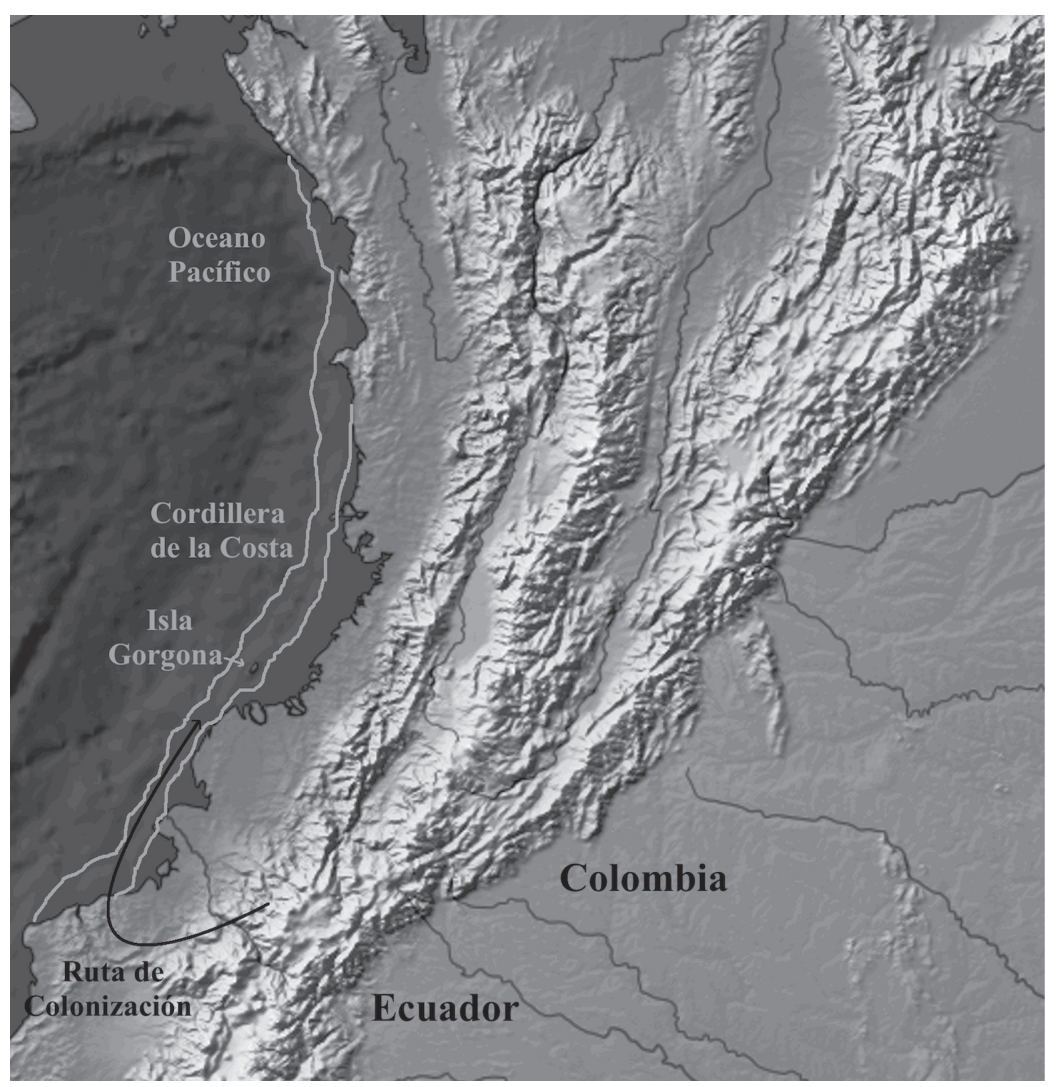

Fig. 4. Ruta hipotética de colonización de C. brevicauda a el Parque Nacional Natural Isla Gorgona. La reconstrucción de la Cordillera de la Costa se basó en Acosta (1986).

Fig. 4. Hypothesized route for the colonization of C. brevicauda to Gorgona National Park. Reconstruction of Cordillera de la Costa was based on Acosta (1986).

evaluadores por sus importantes comentarios y sugerencias que contribuyeron a mejorar la calidad del manuscrito.

\section{RESUMEN}

La identidad de la especie de Carollia (Chiroptera: Phyllostomidae) en el Parque Nacional Natural Gorgona ha sido controvertida debido a la alta similitud entre $C$. perspicillata y C. brevicauda. C. perspicillata es la especie con mayor probabilidad de habitar en la isla debido a que actualmente se distribuye en las tierras bajas de la costa pacífica colombiana, mientras $C$. brevicauda es una especie predominantemente de tierras altas. Con el objetivo de resolver la controversia acerca de la especie del género Carollia que habita la isla, se realizaron análisis morfométricos con base en característica de cráneos y mandíbulas de especímenes de las dos especies y del PNN Gorgona.
Los resultados evidenciaron que, contrario a lo esperado, esta población insular es morfológicamente más similar a C. brevicauda. Evidencia biológica para diferentes grupos de organismos sugiere que Gorgona puede haber estado conectada a los andes y las zonas bajas del pacífico del sur de Colombia-norte de Ecuador en el pasado. Por otra parte, existe evidencia geológica que muestra que Gorgona es la parte más alta de una cordillera (Cordillera de la Costa) que se encuentra sumergida actualmente, pero que probablemente fue parte del continente durante el Pleistoceno. Por lo tanto, con base en esta evidencia biológica y geológica se plantea la hipótesis de una ruta de colonización de C. brevicauda a Gorgona desde los Andes a través de la Cordillera de la Costa. Este proceso pudo facilitarse por el descenso marino durante las glaciaciones del Pleistoceno.

Palabras clave: dispersión de murciélagos, morfología craneal, colonización de islas, biogeografía, taxonomía de murciélagos. 


\section{REFERENCIAS}

Acosta, C. E. (1986). Tectónica de fracturas del suroeste de Colombia. Geología Colombiana, 135-148.

Alberico, M. (1986a). Los mamíferos. In H. v. Prahl \& M. Alberico (Eds.), Isla de Gorgona (pp. 191-209). Fondo de promoción de la cultura del Banco Popular, Bogotá, Colombia.

Alberico, M. (1986b). Biogeografía terrestre. In H. v. Phral \& M. Alberico (Eds.), Isla de Gorgona (pp. 223-245). Fondo de promoción de la cultura del Banco Popular, Bogotá, Colombia.

Alberico, M. (1987). Los Mamíferos. In J. I. Borrero (Ed.), Gorgona (pp. 69-78). Fundación Mejor Ambiente, Cali, Colombia.

Ayala, S., Carvajal, H., Caro, F. \& Castro, F. (1979). Los Saurios de La isla de Gorgona. In H. v. Prahl, F. Guhl \& M. Grögl (Eds.), Gorgona (pp. 219-241). Universidad de Los Andes, Bogotá, Colombia.

Baker, R. J., Solari, S. \& Hoffmann, F. G. (2002). A new central American species from the Carollia brevicauda complex. Occasional Papers, Museum of Texas Tech University, 1-12.

Bolker, B. M. (2008). Ecological models and data in R. Princeton University Press, Princeton, New Jersey, USA.

Cadena, A., Gómez-Laverde, M., Andrade, G. \& Peñuela, A. (1990). Notas sobre la fauna de murciélagos de Gorgona. In J. Aguirre \& J. O. Rangel (Eds.), Biota y Ecosistemas de Gorgona (pp. 236-243). Fondo FEN, Bogotá, Colombia.

Gansser, A. (1950). Geological and petrographic notes on Gorgona island in relation to North-Western South America. Schweizerische Mineralogische und Petrographische Mitteilungen, 30: 219-237.

Haffer, J. (1970). Geologic climatic history and zoogeographic significance of the Urabá region in North Western Colombia. Caldasia, 10: 603-636.

Hedges, L., Gurevitch, J. \& Curtis, P. (1999). The metaanalysis of response ratios in experimental ecology. Ecology, 80: 1150-1156.

Heywood, V. H. \& Watson, R. T. (1997). Global biodiversity assessment, Global Environmental Change. Cambridge University Press, Cambridge, New York, USA.

Hoffmann, F. G. \& Baker, R. J. (2003). Comparative phylogeography of short-tailed bats (Carollia: Phyllostomidae). Moleculary Ecology, 12: 3403-3414.

Lachenbruch, P. A. \& Mickey, M. R. (1968). Estimation of error rates in discriminant analysis. Technometrics, 10: 1-11.

Lourenço, W. R. \& Flores, E. (1989). Los escorpiones (Chelicerata) de Colombia. I. La fauna de la Isla
Gorgona. Aproximación biogeográfica. Caldasia, 16: 66-70.

Martínez, C. (2001). Primer registro de los géneros Glipodes y Boatia (Coleoptera: Mordellidae) para Colombia. Entomotropica, 16: 199-201.

McCune, B. \& Grace, J. B. (2002). Analysis of ecological communities. MjM Software Design, Gleneden Beach, Oregon, USA.

McLellan, L. J. (1984). A morphometric analysis of Carollia (Chiroptera: Phyllostomidae). American Museum Novitates, 2791: 1-35.

McLellan, L. J. \& Koopman, K. F. (2007). Subfamily Carolliinae. In L. A. Gardner (Ed.), Mammals of South America, Vol. 1: Marsupials, Xenarthrans, Shrews, and Bats. The University of Chicago Press, Chicago, USA.

Muñoz, J., Cuartas-Calle, C. A. \& González, M. (2004). Se describe una nueva especie de murciélago del género Carollia Gray, 1838 (Chiroptera: Phyllostomidae) de Colombia. Actualidades Biológicas, 80-90.

Nakagawa, S. \& Cuthill, I. C. (2007). Effect size, confidence interval and statistical significance: a practical guide for biologists. Biological reviews of the Cambridge Philosophical Society, 82: 591-605.

Nygren, W. E. (1950). Bolivar geosyncline of northwestern South-America. AAPG Bulletin-American Association of Petroleum Geologists, 34: 1998-2006.

Owen, J. G., Schmidly, D. J. \& Davis, W. B. (1984). A morphometric analysis of three species of Carollia (Chiroptera, Glossophaginae) from Middle America. Mammalia, 48: 85-93.

Pacheco, V., Solari, S. \& Velazco, P. M. (2004). A new species of Carollia (Chiroptera: Phyllostomidae) from the Andes of Peru and Bolivia. Occasional Papers, Museum of Texas Tech University, 236: 1-15.

Pavan, A. \& Martins, F. (2011). Patterns of diversification in two species of short-tailed bats (Carollia Gray, 1838): the effects of historical fragmentation of Brazilian rainforests. Biological Journal of the Linnean Society, 102: 527-539.

Pine, R. H. (1972). The bats of the genus Carollia [Monograph], Bulletin-Texas Agricultural Experimental Station, 8: 1-125.

Presley, S. J., \& Willig, M. R. (2008). Composition and structure of Caribbean bat (Chiroptera) assemblages: effects of inter-island distance, area, elevation and hurricane-induced disturbance. Global Ecology and Biogeography, 17: 747-757.

R Development Core Team. (2012). R: A language and environment for statistical computing. R Foundation for Statistical Computing, Vienna, Austria.

Silva-Taboada, G. (1974). Fossil Chiroptera from cave deposits in central Cuba, with description of two new 
species (Genera: Pteronotus and Mormoops) and the first West Indian record of Mormoops megalophylla. Acta Zoologica Cracoviensia, 19: 33-73.

Solari, S. \& Baker, R. J. (2006). Mitochondrial DNA Sequence, Karyotypic, and morphological variation in the Carollia castanea species complex (Chiroptera: Phyllostomidae) with Description of a New Species. Occasional Papers, Museum of Texas Tech University, 254: 1-16.

Thomas, O. (1926). On mammals from Gorgona Island, with the description of a new sloth. Annals and Magazine of Natural History, 17: 309-311. von Prahl, H. (1983). Hypolobocera gorgonensis sp. nov. (Crustacea: Brachyura: Pseudothelphusidae) un nuevo cangrejo de agua dulce de la Isla de Gorgona Colombia. Cespedesia, 12: 105-110.

York, H. a. \& Papes, M. (2007). Limiting similarity and species assemblages in the short-tailed fruit bats. Journal of Zoology, 273: 249-256.

Zurc, D. \& Velasco, P. (2010). Análisis morfológico y morfométrico de Carollia colombiana Cuartas et al., 2001 y C. monohernandezi Muñoz et al., 2004 (Phyllostomidae: Carollinae) en Colombia. Chiroptera Neotropical, 16: 567-572. 
